\title{
Non-destructive analysis of anthocyanins in cherries by means of Lambert-Beer and multivariate regression based on spectroscopy and scatter correction using time-resolved analysis
}

\author{
Manuela Zude ${ }^{\mathrm{a}, *}$, Michael Pflanz ${ }^{\mathrm{a}}$, Lorenzo Spinelli ${ }^{\mathrm{b}}$, Carsten Dosche ${ }^{\mathrm{c}}$, Alessandro Torricelli ${ }^{\mathrm{d}}$ \\ ${ }^{a}$ Leibniz Institute for Agricultural Engineering Potsdam-Bornim (ATB), Dept. Horticultural Engineering, Max-Eyth-Allee 100, 14669 Potsdam-Bornim, Germany \\ ${ }^{\mathrm{b}}$ Istituto di Fotonica e Nanotecnologie - Consiglio Nazionale per le Ricerche (IFN-CNR), Piazza Leonardo da Vinci, 32, Milan, Italy \\ ${ }^{\mathrm{C}}$ University of Potsdam, Dept. Physical Chemistry, Karl-Liebknecht-Str. 24-25, Potsdam-Golm, Germany \\ ${ }^{\mathrm{d}}$ Politecnico di Milano - Dipartimento di Fisica, Piazza Leonardo da Vinci, 32, Milan, Italy
}

\section{A R T I C L E I N F O}

\section{Article history:}

Received 19 December 2009

Received in revised form 31 August 2010

Accepted 30 September 2010

Available online 28 October 2010

Keywords:
Cherry
DTOF
Effective pathlength
Fruit maturity
Lambert-Beer
NIR
Non-invasive
Pigments
PLS
Ripeness
Sensor fusion
Spectroscopy
Time-resolved spectroscopy
TIRF
Vis
Scattering

\begin{abstract}
A B S T R A C T
In high-value sweet cherry (Prunus avium), the red coloration - determined by the anthocyanins content - is correlated with the fruit ripeness stage and market value. Non-destructive spectroscopy has been introduced in practice and may be utilized as a tool to assess the fruit pigments in the supply chain processes. From the fruit spectrum in the visible (Vis) wavelength range, the pigment contents are analyzed separately at their specific absorbance wavelengths.

A drawback of the method is the need for re-calibration due to varying optical properties of the fruit tissue. In order to correct for the scattering differences, most often the spectral intensity in the visible spectrum is normalized by wavelengths in the near infrared (NIR) range, or pre-processing methods are applied in multivariate calibrations.

In the present study, the influence of the fruit scattering properties on the Vis/NIR fruit spectrum were corrected by the effective pathlength in the fruit tissue obtained from time-resolved readings of the distribution of time-of-flight (DTOF). Pigment analysis was carried out according to Lambert-Beer law, considering fruit spectral intensities, effective pathlength, and refractive index. Results were compared to commonly applied linear color and multivariate partial least squares (PLS) regression analysis. The approaches were validated on fruits at different ripeness stages, providing variation in the scattering coefficient and refractive index exceeding the calibration sample set.

In the validation, the measuring uncertainty of non-destructively analyzing fruits with Vis/NIR spectra by means of PLS or Lambert-Beer in comparison with combined application of Vis/NIR spectroscopy and DTOF measurements showed a dramatic bias reduction as well as enhanced coefficients of determination when using both, the spectral intensities and apparent information on the scattering influence by means of DTOF readings. Corrections for the refractive index did not render improved results.
\end{abstract}

(C) 2010 Elsevier Ltd. All rights reserved.

\section{Introduction}

Assuring healthy human nutrition and improving the economic success of farmers producing fresh fruit and vegetables are priority targets in the context of current global changes. There is general consensus that especially in agriculture new innovative technologies are needed for appropriate process management. In presently developing technologies, optical methods in particular are principally suitable for onsite analysis. Spectroscopy in near infrared (NIR) as well as visible (Vis) wavelength ranges can serve as a feasible tool for non-invasive fruit analysis due to available miniaturized light sources as well as accurate, robust, and inexpensive

\footnotetext{
* Corresponding author.

E-mail address: zude@atb-potsdam.de (M. Zude).
}

spectrophotometer modules. The compounds have been implemented in high-end colorimeters, handheld units for spectroscopic analyses, and sorting lines (Ozaki et al., 2006; Molto and Blasco, 2009). Based on the physics of NIR spectroscopy, a detailed review for agricultural and food products was presented by Birth and Hecht (1987) and, more recently, focusing on the initial data processing in fruit analyses, by Nicolai et al. (2007).

The Vis part of the spectrum provides information on the pigment contents. Spectra obtained non-destructively from the fruit sample are frequently analyzed in terms of color using various color spaces. Since this paper uses sweet cherry as a model fruit, the literature was searched for correlations of spectral readings respective to fruit quality and ripeness stage. Color readings for grading fruits are evaluated regarding the external appearance recognized by the consumer. High correlation of color values in $L^{*} a^{*} b^{*}$ color 
space and the anthocyanin contents of cherries were presented in recent papers (Serrano et al., 2005; Goncalves et al., 2007; Manganaris et al., 2007; Serrano et al., 2009). It was shown that variations of fruit pigment composition in different cultivars and colors correlate with the anthocyanins content and anti-oxidative capacity, with higher values in cultivars with visually dark appearance (Karlidag et al., 2009). Ripening-related enhanced color appearance and corresponding anthocyanin content resulted in decreased values of $L^{*}, a^{*}$, and $b^{*}$ during fruit development, shortly before harvest time at the tree (Kovacs et al., 2009) as well as in postharvest (Serrano et al., 2009).

However, it might be assumed that the apparent color data can be influenced by other fruit pigments than anthocyanins such as chlorophylls and carotenoids, showing absorption at coinciding wavelengths. As an alternative, specific indices or whole spectra analyses have been introduced in practice and may serve as a more reliable method for measuring the cultivar- and maturity-related pigment development. From the fruit spectrum in the visible wavelength range, the pigment contents are analyzed separately at their specific absorbance wavelengths (Knee, 1972; Nagata and Yamashita, 1992; Pflanz and Zude, 2008).

Using Vis spectral readings of intact horticultural produce, the specific absorption characteristics can provide sensitive information on the changes in pigment contents. The data point to physiological stages such as the chlorophyll-related ripeness in apple (Merzlyak et al., 1999; Zude, 2003; Peirs et al., 2005; Solovchenko et al., 2005; Herold et al., 2005), and can be used to assess the nutritional value of the product such as the content of native carotenoids in carrots (Zude et al., 2007). In this field of pigment detection, research in canopy remote sensing rendered the initial impulses (Penuelas et al., 1995; Gamon et al., 1997; Richardson et al., 2002). Several indices have been established in feasible applications, mainly for chlorophyll analysis, but also anthocyanin readings.

A drawback in the analysis of fresh, rapidly developing produce appears due to changes in the chemical composition as well as texture of the fruits. Researchers as well as developers of commercial Vis/NIRS equipment face difficulties with the robustness of calibration based on apparent spectral intensity, $I_{R}(\lambda)$. In fruit samples, we have to deal with the absorption coefficient at the wavelength under question, $\mu_{a}(\lambda)$ that provides quantitative information on the molecule content under question, as well as multiple scattering during the photon transport in the tissue. It can be assumed that depending on the phenotype of the fruit the scattering coefficient, $\mu_{s}$, as well as the directional anisotropy factor, $g$, are varying. Results from simulations show that in some cases $g$ has a major effect on the optical properties of kiwi fruit at different ripeness stages (Baranyai and Zude, 2009), while in apples the differences in $g$ during fruit development appear marginal (unpublished data). The variation in $\mu_{s}$ is in any case a major factor, influencing the result when applying a calibration model. The resulting need for re-calibrations heavily limits the introduction of the sensors in practice.

The differences in the scattering properties actually have an impact on the effective pathlength, $L^{*} s$, that the photon is traveling in the tissue on its way from the light source to the detector. If the scattering coefficient and, consequently, the effective pathlengths would be known, the Lambert-Beer law might be applied for pigment, $c_{i}$, analyses in intact fruit, such as presently carried out in the laboratory on fruit extracts:

$\log \left[100 / I_{R}(\lambda)\right]=\sum c_{i} \varepsilon_{i} L^{*} S$

It is accepted to estimate the effective pathlength of photons traveling through a sample by means of the reduced scattering coefficient, $\mu_{s}^{\prime}=1 / L^{*} s$ (Cubeddu et al., 1999). While it appears difficult to obtain information on $\mu_{s}$ and $g$ separately, the reduced scattering coefficient, $\mu_{s}^{\prime}=(1-g) \mu_{s}$, can be measured by means of distribution of time of flight (DTOF) readings.

The time-resolved DTOF readings consist of injecting a short (picosecond) light pulse into the fruit to be characterized, while the transmitted or reflected photons are detected in a certain distance from the injector after traveling in the pericarp of the fruit. Attenuation and delay in pulse shape are due to the absorption and scattering events occurring into the sample, and an estimate of the optical parameters can be derived, based on a suitable model of light propagation, i.e. diffusion theory or Random Walk model (Patterson et al., 1989; Contini et al., 1997; Cubeddu et al., 1999). Applying DTOF the $\mu_{a}$ can be uncoupled from the $\mu_{s}$ (Patterson et al., 1989; Jacques, 1989; Arridge et al., 1992). A pre-assumption for application of DTOF in fruits is that the $\mu_{s}>>\mu_{a}$ and the sample is large enough to avoid photon loss at the boundary of the sample. This is certainly not the case in fruits. However, predictions of the fruit SSC (Tsuchikawa and Hamada, 2004) and maturity-related chlorophyll content (Eccher Zerbini et al., 2006) were approached already by means of DTOF analyses.

Coming back to our problem of varying pathlength in the fruit samples and resulting perturbation in non-destructive analysis. It appears to be reasonable - even with expected high uncertainty of DTOF in cherries - to propose that the scattering influence on the apparent intensities in the fruit spectra can be corrected: knowing the spectral intensities at the specific absorption passband of the pigments as well as the effective pathlength by means of empirical DTOF data. Subsequently, a robust calibration on a certain pigment should be possible.

As an additional uncertainty, the refractive index of the sample has an impact on the apparent data from DTOF analysis due to varying boundary conditions (Contini et al., 1997). During fruit development the refractive index is changing and the influence of the variation in the refractive index on the calculation of the effective pathlength was not clear. Therefore, the non-destructive reading of the refractive index on the fruit material was approached as an additional step in sensor fusion in the present study. The total internal reflectance fluorescence (TIRF) microscopy was selected for this purpose, which is an emerging and effective technique for examining properties in the living cell structure (Oheim and Schapper, 2005). The method was chosen due to the opportunity for non-destructive readings. The study was aimed at evaluating an improvement in pigment analysis by means of the application of the actual refractive index.

The approach was compared with the established corrections by means of indices or multivariate data pre-processing. Indices use specific absorption passbands of the molecule corrected by NIR passband in the so-called "optical window". In the optical window almost no plant compound absorbs photons, and therefore, all variation appears due to scattering differences. Alternatively, adapted data pre-processing in whole-spectra analyses can be applied such as multiplicative signal correction (MSC), standard normal variates (SNV), or working on derivatives. More recently orthogonal signal correction and further developed method as direct orthogonal signal correction were proposed for removing information not relevant to the absorption correlated with the molecule content under question. While having the advantage of being easy to apply methods of dealing with the problem, the approaches do not characterize the phenomenon itself. Furthermore, re-calibration is still required, if the calibration data-set does not capture all variation appearing the samples for prediction.

In the present study, cherries were chosen as commercially interesting samples. New cultivars with enhanced fruit size and appealing coloration have been released, which are sold at extremely high prices. Such new high-value cultivars raise questions regarding the processes of optimum harvest date determination, 
selective harvesting, and postharvest handling - questions that might be answered by means of non-invasive analyses in the supply chain, when a feasible method becomes available.

The approach carried out, was aimed at improving the robustness of Vis spectroscopy by means of sensor fusion approach. The influences of varying optical fruit properties on the pigment calibration were corrected (i) with the effective pathlength in the fruit tissue obtained with DTOF readings as well as (ii) with additional data on the boundary conditions of the sample obtained by means of TIRF analysis. In comparison, commonly applied data processing methods were tested such as (iii) normalized index, (iv) multivariate spectra processing,

For testing the approach, data set for calibration and slightly different data set for validation in similar ranges as it appears in practice were required. The cherries subjected to the experiments were harvested on the same trees, but in earlier and more advanced ripeness stages. Such conditions would occur for all nondestructive equipment applied in the field.

\section{Material and methods}

\subsection{Fruit material and quality analysis}

Sweet cherry, Prunus avium 'Schneiders späte Knorpel', was analyzed immediately after harvesting in different ripeness stages: unripe, intermediates 1-3 (im1-3), and ripe. Fruits were subjected to chemical pigment analysis as well as Vis/NIR spectroscopy, DTOF, and TIRF readings. Forty-five fruits were harvested on one day at five ripeness stages $(n=9)$ and analyzed within $48 \mathrm{~h}$. Acidity and fruit size were measured.

The anthocyanins and carotenoids contents of the exocarp and mesocarp of cherry were analyzed wet-chemically by spectrophotometry after acetone/diethyl ether extraction. In the ether fraction the carotenoids were analyzed, while the anthocyanins accumulated in the polar phase. Standards of lutein, $\alpha$-carotene, and $\beta$-carotene were applied for carotenoids analyses and the anthocyanins were expressed as cyanidin (Carl Roth GmbH, Germany) equivalents, each pigment by subjecting the spectra of extracts to multiple linear regression based on the standard solutions and minimizing the error when recalculating the sum spectra of extracts (Pflanz and Zude, 2008).

\subsection{Non-destructive sensing}

Colorimetric readings (CM-2600d/2500d, Minolta, Japan) were recorded by means of $L^{*} a^{*} b^{*}$ (D65) values that were calculated with the instrument software according to CIE standards.

The fruit spectra were recorded $(400-850 \mathrm{~nm})$ in diffuse reflectance mode by means of a scanning dual beam spectrophotometer unit (Lambda 950, Perkin Elmer, USA) on referenced (100\% Spectralon, Labsphere Ltd., North Sutton, U.S.A.) wavelength intensities.

The rededge, inflection point of chlorophyll absorption peak determined by $f^{\prime}(660-710 \mathrm{~nm})=0$, and a normalized index, $\left(I_{620}-I_{780}\right) /\left(I_{620}+I_{780}\right)$, were calculated on the spectral intensities, $I_{R}(\lambda)$ from Vis/NIR readings. The rededge is accepted to correct for scattering and resulting vertical shifts of the spectra due to the calculation on the spectra derivatives. The index was calculated on the edge of anthocyanidin absorption passband and a passband in the "optical window" to correct for varying scattering properties.

A system to measure DTOF in transmittance geometry was used, working at 780 and $670 \mathrm{~nm}$, based on a pulsed laser diode (PDL800, PicoQuant GmbH, Germany) with $80 \mathrm{MHz}$ repetition frequency, 100 ps duration, and $1 \mathrm{~mW}$ average power, with a compact photomultiplier (R5900U-L16, Hamamatsu Photonics, Japan) and an integrated PC board for time-correlated single photon counting (SPC130, Becker\&Hickl GmbH, Germany). The width of the instrumental response function (IRF) was $<160$ ps (Torricelli, 2009). Acquisition time was set to $1 \mathrm{~s}$ per fruit. The values of reduced scattering coefficient and absorption coefficient were obtained from fitting the experimental data with a standard solution of the diffusion approximation to the transport equation for a semi-infinite homogeneous medium. The diffusion coefficient was taken to be independent of the absorption properties of the medium. The theoretical curve was convoluted with the instrumental response function, and was normalized to the area of the experimental curve (Cubeddu et al., 1999). The procedure was described in detail in former studies about fresh horticultural products (Cubeddu et al., 1999; Torricelli, 2009).

TIRF measurements were performed using a confocal imaging system (Infinty 3, Visitron Systems, Germany) equipped with an additional TIRF port. For excitation, the krypton laser line at $647 \mathrm{~nm}$ of an argon-krypton ion laser (Innova 90, Coherent, CA, USA) was applied. The excitation light was coupled into the intact cherry sample through a $100 \times$ oil immersion objective. The incident angle for determination of the threshold angle for total reflection could be adjusted with a micrometer screw. The threshold position of the screw and related incident angle were calibrated for the refractive index with sucrose solutions of known refractive index containing $\sim 10^{-5} \mathrm{M}$ Cy5 (Cy5 excitation and emission maximum at 649 and $670 \mathrm{~nm}$, respectively, quantum yield is 0.28 ). The refractive index of the calibration solutions was checked with a standard laboratory refractometer (Carl Zeiss, Germany). Calibration, $y=707.65 x-632.58, r^{2}=0.99$, on the apparent refractive index measured in sucrose solutions (0-18.2\%Brix). The calibration was tested on intact cherries. The prediction referenced by means of destructive refractometric readings of the squeezed fruit juice was $r^{2}=0.77$.

\subsection{Calculation of effective pathlengths}

The pathlength, $L$, was determined by the penetration depth, $\delta$, (Eq. (2)) calculated based on diffusion theory (Torricelli, 2009), which resulted in an average value of $0.3 \mathrm{~cm}$.

$\delta(780 \mathrm{~nm})=1 / \mu_{\text {att }}=1 / \sqrt{3 \mu_{a}\left(\mu_{a}+\mu_{s}^{\prime}\right)}$

The effective pathlength, $L^{*} s_{1}$ was calculated by Eq. (3), where $c$ is the speed of light in vacuum $(c \approx 0.03 \mathrm{~cm} / \mathrm{ps})$, and $N$ is the refractive index of the medium. For calculating $L^{*} s_{1}$ the refractive index was assumed constant with $N=1.4$, while for calculating $L^{*} s_{2}$ (Eq. (4)) the actual refractive index was used based on the TIRF readings.

$$
L^{*} s_{1}(780 \mathrm{~nm})=<t>c / 1.4
$$$$
L^{*} s_{2}(780 \mathrm{~nm})=<t>\cdot c / N
$$

\subsection{Calibrations}

For calibration and validation (Fig. 1) the data sets were split in two blocks, one block providing the values for unripe and half of the intermediate samples and the second block containing the values of the remaining intermediates and ripe fruits.

Calibrations were carried out by means of linear regression either using the color values, index, and results from the application of Lambert-Beer law, using the three pathlengths or by means of multivariate partial least squares (PLS1) calibration models. The latter were built in Matlab (version 7.6.0.324 R2008a, The MathWorks, USA) on raw data of the Vis/NIR spectra pre-processed by means of multiple signal correction (MSC) and second derivative. The optimum numbers of latent variables (LV) were selected on 
the basis of minimizing the root mean square error by selecting the first minimum in cross-validation with leave-one-out routine.

The measuring uncertainty was separated into two independent estimation errors:bias $(e b)$ and remaining root mean square error (rmse). Percentage estimation errors were obtained by dividing by the mean of actual value. The errors, correlation coefficient $(r)$ and coefficient of determination $\left(r^{2}\right)$ were calculated in Matlab.

\section{Results and discussion}

\subsection{Fruit development and fruit spectra}

In unripe cherries and fruits in slightly enhanced ripeness stage (im1), chlorophyll was noticed in the fruit spectra around $680 \mathrm{~nm}$, but was at the detection limit in the chemical analyses (Fig. 2A). The rededge (660-710 nm), applied as chlorophyll estimator in precision agriculture (Richardson et al., 2002) and fruit analysis (Zude, 2003), indicated decreasing chlorophyll contents providing interesting data on the fruit development. However, in more advanced ripeness stages the chlorophyll already disappeared.

Cherries of all five ripeness stages showed spectral variation at the blue-green passband with a sharp intensity increase appearing in the range of $550-650 \mathrm{~nm}$ (Fig. 2A). The region of enhanced reflectance intensities was shifted further in the red wavelength range the more red pigmentation occurred. Consequently, the intensity change provides a rough marker for the fruit ripeness stage. The edge appearing at the passband of $620 \mathrm{~nm}$ was used for further data processing. The non-invasively measurable data are already of practical interest for the production and harvest manager in an orchard, since fruits at advanced ripeness stage exhibiting more intense pigmentation can be selected. In practice, the variation of fruits appearing at the same time on the tree point to the potential value-adding by means of selective harvesting such as carried out in the apple production. Such suggestion appears reasonable since the market price increases exponentially mainly with the ripeness stage and quality regarding the fruit sweetness expressed as soluble solids content (SSC) and each $\mathrm{mm}$ in fruit size (Table 1). Resulting, non-invasive readings can help to make the processes, for instance the harvest management, more efficient.

In cherry, the red pigmentation is caused by the anthocyanins and carotenoids contents. Even if the cumulative increase of red pigments provides some information, a more specific data analysis of the fruit spectra would be helpful for characterizing the fruit development onsite by means of non-invasive readings. Comparing the evolution of red pigment contents, the $\alpha$-carotene decreased, while the total carotenoids did not show consistent differences during fruit development. The anthocyanin - here expressed as cyanidin equivalents - content was enhanced with higher ripeness stages (Fig. 2B). Although the $\alpha$-carotene and the anthocyanins changed significantly with the fruit development, only the anthocyanins were studied further due to their higher contents and, therefore, more pronounced contribution to the changes in the fruit spectra.

\subsection{Calculation of the effective pathlength}

A robust and non-invasive analysis of the pigments was approached for characterizing the fruit ripeness stage. The pigment analysis was carried out by applying Lambert-Beers law directly on the intensity data, $I_{R}(620 \mathrm{~nm})$ obtained with Vis/NIR remittance readings and the constant pathlength, $L=0.3 \mathrm{~cm}$. The constant pathlength (Eq. (2)) was derived from diffusion theory, which is obviously underestimating the apparent light penetration. The suitability of different theories and optical geometries (Arridge et al., 1992) has been explored by different work groups. However, the diffusion theory is widely used and applied in the present work for principally testing our approach.

Additionally, the effective pathlength was estimated by means of DTOF. The mean time-of-flight $(<t>)$ was derived from the distribution of incoming photons recorded (Fig. 3A). The DTOF was measured for wavelength $\lambda=780$ and $\lambda=670 \mathrm{~nm}$. The readings from $670 \mathrm{~nm}$ gave a weak signal in ripe cherries due to high absorption coefficients of cyanidins in more advanced ripeness stages. Resulting, the data of $780 \mathrm{~nm}$ (Fig. 3B) were used further for calculating the effective pathlength, $L^{*} s$ (Eqs. (3) and (4)).

For biological tissue $N=1.4$ is typically used as an estimation. However, the cherries developed enhanced values of $N$ and corresponding SSC during fruit ripening - the latter ranging from 9.9 to $20.1^{\circ}$ Brix. Since a possible influence on the calculation should be neglected in the present study, the actual refractive index was measured non-destructively by means of TIRF. Structures from cell compounds appeared in the image (Fig. 4A), when the fluorescence appeared in the evanescent field and total internal reflection occurred. According to Snell's law, the refractive index was derived. The angle of total reflectance was sensitively recorded by means of histograms of the images (Fig. 4B). At the threshold angle the excitation light no longer coupled into the sample via evanescent field, but directly, leading to a dramatic increase in intensity und thus sudden light overexposure of the image.

Based on the calibration with standard solution, the refractive index at wavelength $647 \mathrm{~nm}$ was non-destructively measured. The $N$ changed over the different ripeness stages (Fig. 5), and was tested in calculating the effective pathlength, $L^{*} s_{2}$ (Eq. (4)).

Resulting, in the present study, the constant value of $N=1.4$ for calculating the effective pathlength, $L^{*} s_{1}$, and the data obtained by means of non-invasive TIRF for calculating the effective pathlength, $L^{*} s_{2}$, were applied. Accordingly to the change in refractive index, the effective pathlength changed, resulting in 9.64\% higher values when applying the refractive index estimated from TIRF readings (Table 2).

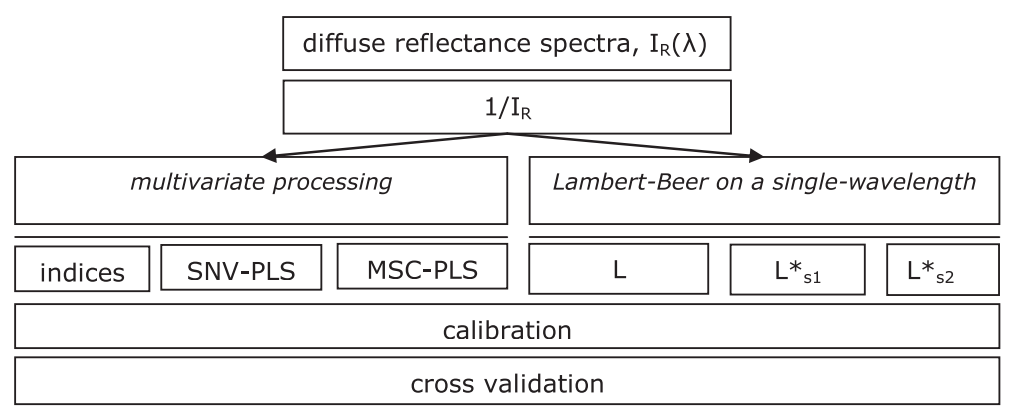

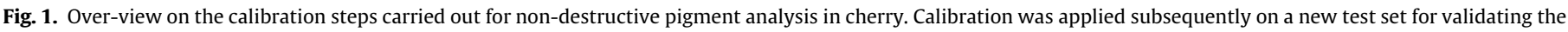
approaches (Tables 3 and 4). 
Table 1

Correlation table of fruit data obtained on five ripeness stages $(n=43)$ of sweet cherry.

\begin{tabular}{|c|c|c|c|c|c|c|c|c|c|c|c|}
\hline & Size & SSC & Acidity & Carotenoids & Cyanidin & Rededge & $L^{*}$ & $a^{*}$ & $b^{*}$ & $<t>$ & $N$ \\
\hline Size & 1 & 0.02 & 0.30 & 0.07 & 0.28 & 0.17 & -0.05 & 0.00 & -0.07 & 0.38 & 0.14 \\
\hline SSC & & 1 & -0.22 & -0.12 & 0.76 & -0.84 & -0.68 & -0.69 & -0.74 & -0.46 & 0.74 \\
\hline Acidity & & & 1 & 0.61 & -0.55 & 0.48 & 0.40 & 0.57 & 0.53 & 0.65 & -0.32 \\
\hline Carotenoids & & & & 1 & -0.34 & 0.32 & 0.18 & 0.34 & 0.30 & 0.53 & 0.02 \\
\hline Cyanidin & & & & & 1 & -0.96 & -0.53 & -0.75 & -0.69 & -0.64 & 0.68 \\
\hline Rededge & & & & & & 1 & 0.54 & 0.74 & 0.69 & 0.64 & -0.73 \\
\hline$L^{*}$ & & & & & & & 1.00 & 0.66 & 0.90 & 0.50 & -0.78 \\
\hline$a^{*}$ & & & & & & & & 1.00 & 0.91 & 0.54 & -0.52 \\
\hline$b^{*}$ & & & & & & & & & 1.00 & 0.56 & -0.70 \\
\hline$\langle t>$ & & & & & & & & & & 1 & -0.44 \\
\hline
\end{tabular}
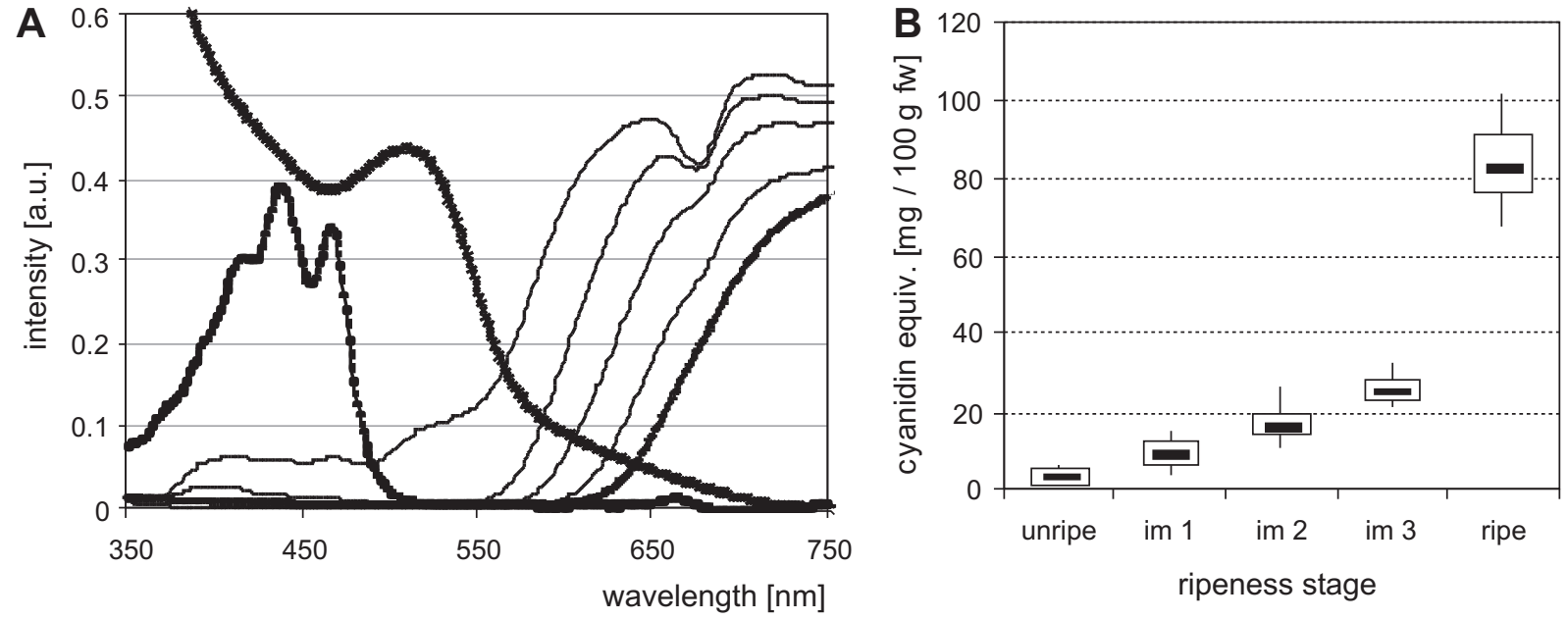

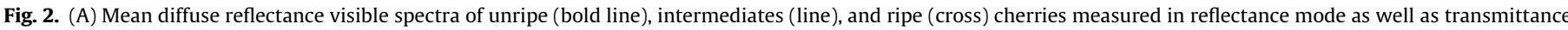

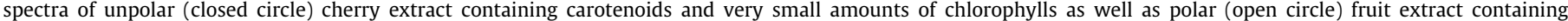
anthocyanidins. (B) Boxwhisker plot with average shown as line symbol of the cyanidin contents of fruits at five ripeness stages.
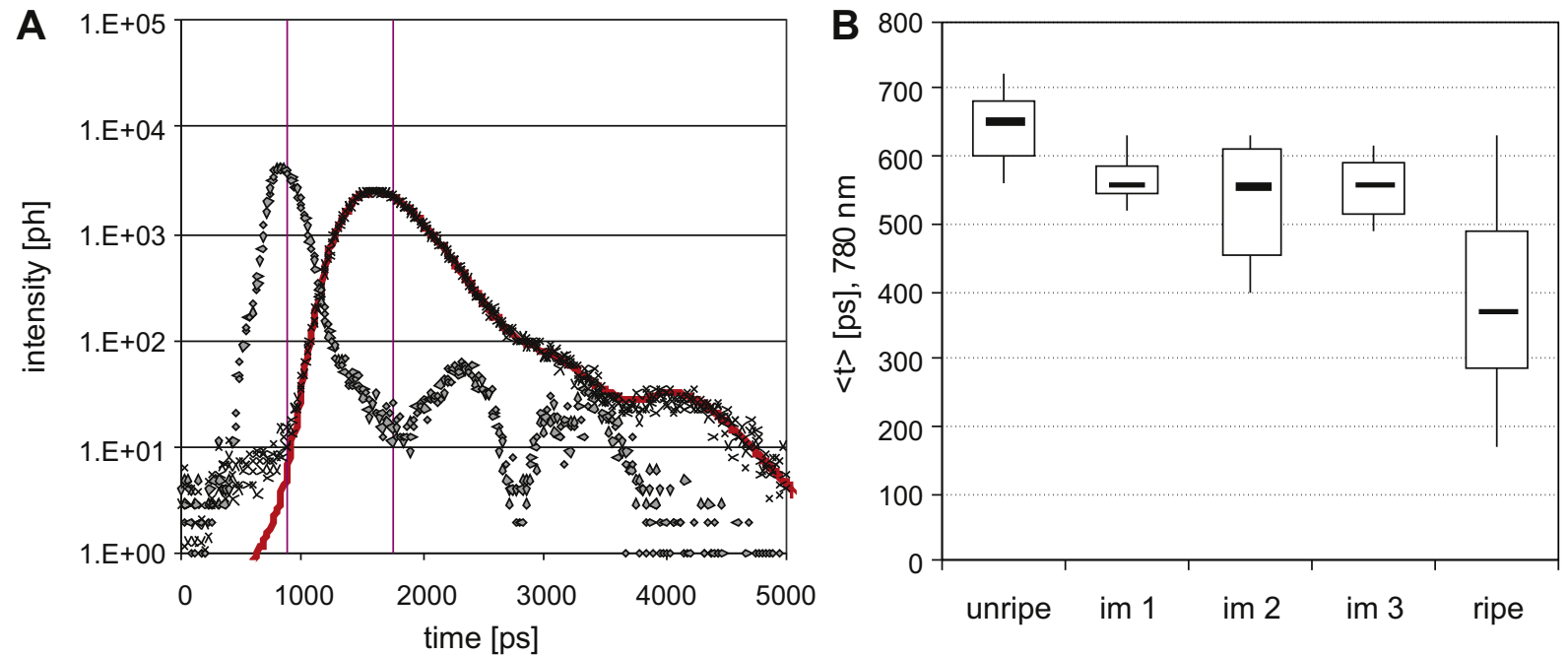

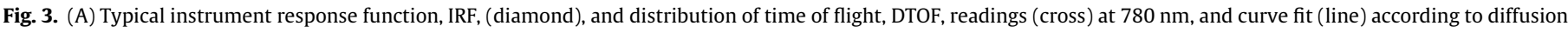

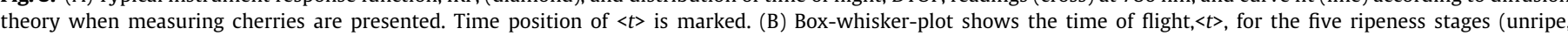
intermediates im $1-3$, ripe) studied.

\subsection{Calibration on cyanidin contents based on Lambert-Beer}

Simple linear calibrations on the cyanidin contents of cherry fruit were carried out based on color data and the use of a normalized index, calculated on the absorption maxima of pigments and a passband in the optical window to correct for varying scattering properties. Calibration results point to expected highest correlation of $b^{*}$-value and cherry cyanidin contents (Table 3 ).

Loadings of PLS regression analyses showed highest variance of first three and four latent variables around $624 \mathrm{~nm}$ passband 

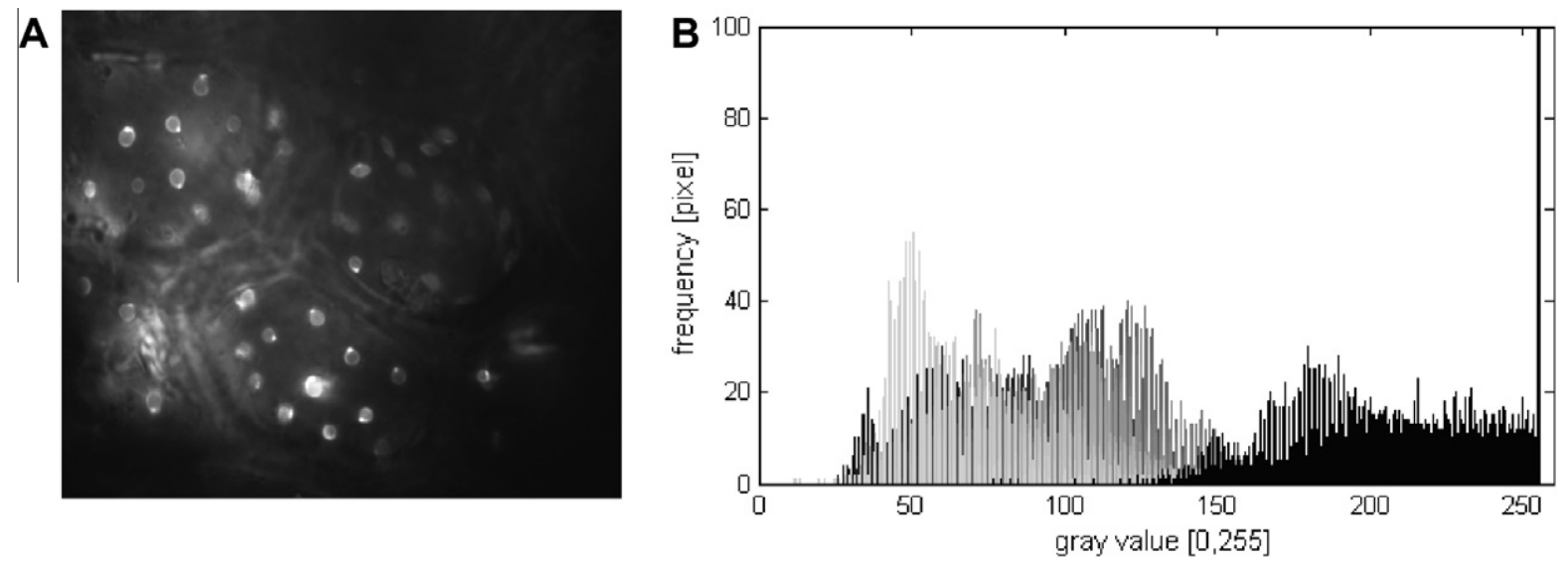

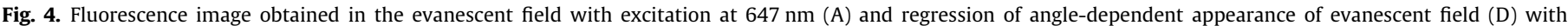
histogram of image obtained in total reflectance (gray columns) and outside evanescent field (black columns) presented in one diagram (B).

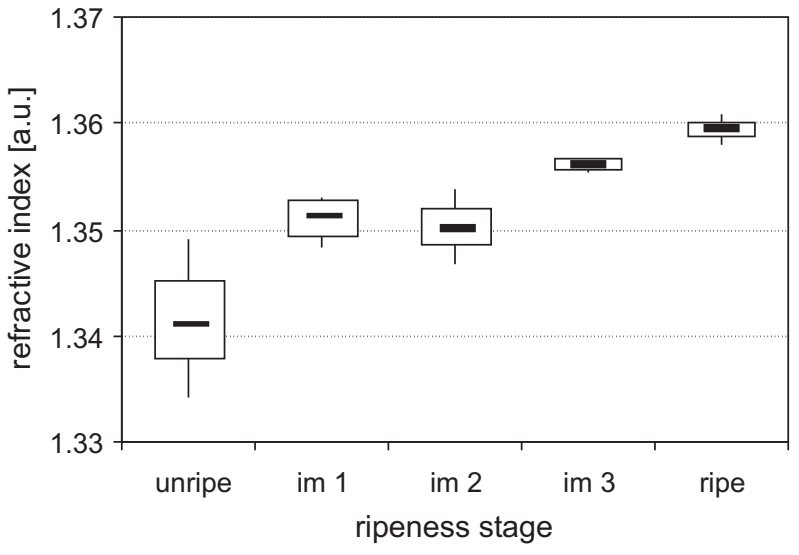

Fig. 5. Refractive index of sweet cherry measured by means of total internal reflection fluorescence (TIRF) readings.

supporting the choice of $I_{R}(620 \mathrm{~nm})$ for calculations. At three latent variables the minimum in cross-validation in leave-one-out mode was reached, capturing variances of $98.69 \%$ and $82.53 \%$ in spectral intensity and cyanidin content, respectively. Increased $r^{2}$-value was found when calculating on 2nd derivative obtained by means of Savitzky-Golay routine with window size of 5 wavelengths and 2nd order polynom (Table 4).

Furthermore, the constant as well as effective pathlengths were subjected to the pigment, $c_{i}$, analyses by means of Lambert-Beer with: $I_{R}(620 \mathrm{~nm})=c_{i} . L^{*} s_{n}(780 \mathrm{~nm})$. The calibration step was carried out on unripe and intermediate (im1-2) cherry fruits. The constant pathlength as well as the effective pathlength, obtained by means of DTOF, assuming a refractive index of 1.4 , and the effective pathlength, calculated by DTOF taking into account the actual refractive index obtained from TIRF readings, resulted in low bias but rather high diffusion errors in all calibrations (Table 4). In former studies (Zude et al., 2008) the method of fusing visible spectra and DTOF data was tested for liquid phantoms mimicking the optical properties of fresh carrot. Lambert-Beer law using a constant pathlength as well as combined application of the intensity at a specific wavelength and the effective pathlength had been resulted in low calibration errors with $r^{2}>0.98$. However, when the two calibrations were applied on phantoms mimicking changes in the scattering properties, validation results of $r^{2}=0.47$ and $r^{2}=0.64$ with reduced bias but also high diffusion error were found, respectively.

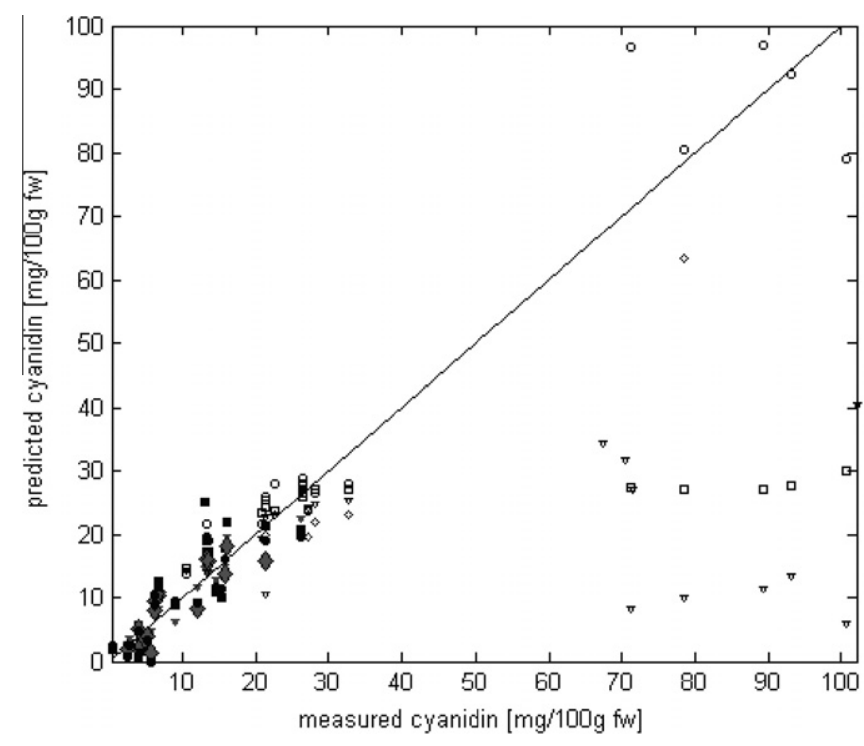

Fig. 6. Scatter plot of measured and predicted values of cyanidin contents in cherry in the calibration (closed symbols) and in the independent test-set validation (open symbols) on fruits in advanced ripeness stages by means of data analyses based on $L^{*} s_{1}$ (circle), $L^{*} s_{2}$ (diamond), and constant $L=0.3$ (square), as well as frequently applied PLS with MSC pretreatment (triangle).

\subsection{Validation results}

Interesting results were found when using the calibration models on samples in ripe and overripe stages - providing variation in the scattering coefficient and resulting effective pathlength (Fig. 3B). The validation point to an increase in $r^{2}$ and obvious bias reduction (Fig. 6) when using the effective pathlength data (Tables 3 and 4). The high coefficients of determination when using the apparent effective pathlengths partly appear due to extremely high variation in the cyanidin contents, exceeding the range in the calibration.

Coefficients of determination in the validation were $r^{2}=0.45$ and $r^{2}=0.92$, comparing the use of spectral intensities from Vis/ NIR readings or combined use of spectral intensities and effective pathlength from DTOF analysis, respectively (Table 4).

Validation based on normalized index gave $r^{2}$-value of 0.25 ; color statistics remained rather stable, while PLS models resulted in dramatically decreased $r^{2}=0.14$. In the validation, the errors appearing when PLS regression analysis on pretreated spectral data was used, can certainly be reduced by means of larger data set used 
Table 2

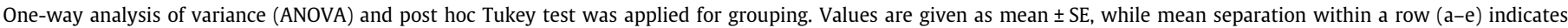
significant differences at 0.05 .

\begin{tabular}{|c|c|c|c|c|c|}
\hline & Unripe & $\operatorname{im} 1$ & $\operatorname{im} 2$ & $\operatorname{im} 3$ & Ripe \\
\hline Cyanidin & $3.52 \pm 1.89 a$ & $9.03 \pm 4.02 \mathrm{a}$ & $15.9 \pm 4.88 \mathrm{a}$ & $32.74 \pm 24.12 b$ & $80.44 \pm 12.8 c$ \\
\hline $\log I_{620}$ & $0.2010 \pm 0.05 a$ & $0.5275 \pm 0.08 b$ & $0.9491 \pm 0.13 c$ & $1.2167 \pm 0.09 d$ & $1.2849 \pm 0.03 \mathrm{ed}$ \\
\hline$<t>$ & $651.82 \pm 55.60 a$ & $556.72 \pm 37.90 a$ & $553.05 \pm 77.99 a$ & $525.57 \pm 109.61 a$ & $388.96 \pm 222.89 b$ \\
\hline$L^{*} s_{1}$ & $13.97 \pm 1.19 a$ & $11.93 \pm 0.81 a$ & $11.85 \pm 1.67 a$ & $11.26 \pm 2.34 a$ & $8.33 \pm 4.78 \mathrm{ba}$ \\
\hline$N$ & $1.3412 \pm 0.010 \mathrm{a}$ & $1.3514 \pm 0.002 b$ & $1.3501 \pm 0.003 b$ & $1.3562 \pm 0.000 b c$ & $1.3598 \pm 0.001 \mathrm{dc}$ \\
\hline$L^{*} s_{2}$ & $15.04 \pm 1.13 a$ & $12.54 \pm 0.99 a$ & $12.23 \pm 1.88 a$ & $12.19 \pm 0.74 a$ & $11.41 \pm 3.94 a$ \\
\hline
\end{tabular}

Table 3

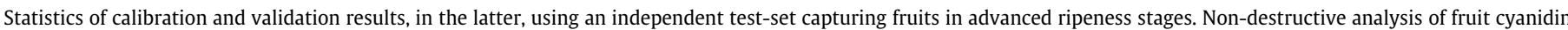
contents was carried out using linear regression by means of color data, $L^{*} a^{*} b^{*}$, and wavelength-specific normalized index, $\left(I_{620}-I_{780}\right) /\left(I_{620}+I_{780}\right)$.

\begin{tabular}{|c|c|c|c|c|}
\hline & $\begin{array}{l}\text { Index } \\
y=0.0268 x+0.358\end{array}$ & $\begin{array}{l}L^{*} \\
y=-1.2494 x+45.551\end{array}$ & $\begin{array}{l}a^{*} \\
y=-1.1681 x+37.546\end{array}$ & $\begin{array}{l}b^{*} \\
y=-0.8665 x+21.198\end{array}$ \\
\hline \multicolumn{5}{|c|}{ Calibration results on unripe and intermediates 1 and $2(n=23)$} \\
\hline$\%$ rmse & 50.927 & 63.323 & 108.857 & 34.950 \\
\hline$\% e b$ & -25.454 & 0.000 & 0.001 & -10.981 \\
\hline$r^{2}$ & 0.581 & 0.517 & 0.270 & 0.592 \\
\hline \multicolumn{5}{|c|}{ Validation uncertainty on intermediate stage 3 and ripe cherries $(n=20)$} \\
\hline$\%$ rmse & 2.065 & 6.254 & 56.711 & 7.262 \\
\hline$\% e b$ & -152.888 & -133.714 & 46.474 & -112.601 \\
\hline$r^{2}$ & 0.246 & 0.363 & 0.670 & 0.600 \\
\hline
\end{tabular}

Table 4

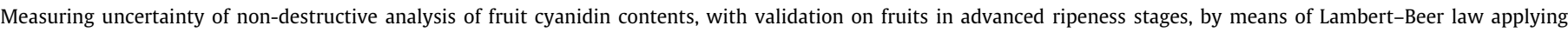
different degree of sensor fusion and resulting pathlengths: $L=0.3, L^{*} s_{1}, L^{*} s_{2}$, as well as from whole spectra partial least squares (PLS) regression analysis.

\begin{tabular}{|c|c|c|c|c|c|}
\hline & $\begin{array}{l}L=\text { constant } \\
y=0.0035 x+0.0097\end{array}$ & $\begin{array}{l}L^{*} S_{1} \\
y=0.0028 x+0.0088\end{array}$ & $\begin{array}{l}L^{*} S_{2} \\
y=0.0044 x+0.0035\end{array}$ & $\begin{array}{l}\text { PLS-model on data pretreated with MSC using } 3 \\
\text { LV }\end{array}$ & $\begin{array}{l}\text { PLS-model on 2nd derivative using } 4 \\
\text { LV }\end{array}$ \\
\hline \multicolumn{6}{|c|}{ Calibration on unripe and intermediates 1 and $2(n=23)$} \\
\hline$\%$ rmse & 36.909 & 39.894 & 28.702 & 28.420 & 14.045 \\
\hline$\% e b$ & -0.530 & 0.619 & -0.904 & 6.024 & 8.413 \\
\hline$r^{2}$ & 0.667 & 0.669 & 0.707 & 0.767 & 0.985 \\
\hline \multicolumn{6}{|c|}{ Validation result on intermediate stage 3 and ripe cherries $(n=20)$} \\
\hline$\%$ rmse & 12.640 & 10.626 & 3.053 & 6.036 & 16.617 \\
\hline$\% e b$ & -33.176 & 4.919 & -8.108 & -138.435 & -113.359 \\
\hline$r^{2}$ & 0.453 & 0.921 & 0.950 & 0.142 & 0.004 \\
\hline
\end{tabular}

in the calibration and further improved by means of different data pre-processing, or by using multiple linear regression. When using the entire data set including unripe to overripe cherries in the calibration with MSC pre-treatment, the statistical results of cross validation in leave-one-out mode were: $r^{2}=0.83$, rmse $=20.58 \%$, and bias was $-14.26 \%$.

However, big data sets capturing all possible changes in the optical properties of a fruit under question are necessary for PLS calibration, but are rarely available in practice. Fruits are changing not only with the ripeness stage, but also from year to year, during tree ontogenesis, according to the production system, and environmental conditions. Consequently, the measurement of the effective pathlength provides an interesting alternative for developing robust calibrations instead of extremely large data sets for multivariate calibration with statistical pre-treatment.

\section{Conclusions}

It was pointed out that (i) the effective pathlength of photons traveling in the fruit is one main influencing factor on the robustness of calibrations, and (ii) it is possible to correct for this varying value. Such conclusion can be assumed, even if the results on the reduced scattering coefficient and the effective pathlength calculated can be seen only as principle proof due to inaccuracy of diffusion theory in modeling highly absorbing tissue, use of the reduced scattering coefficient that does not provide distinct data on $g$ and $\mu_{s}$, small fruit samples which cannot assumed to be infinite, and a marginal wavelength dependence of $\mu$ 's in the relevant wavelengths (780 and $620 \mathrm{~nm}$ ).

Particularly in fresh, rapidly developing produce with changes in the chemical composition as well as texture, the effective pathlength can be used to correct for variation in the scattering coefficient that disturbs the apparent signal of non-destructive analyses in the field.

\section{Acknowledgment}

The research leading to these results has partially received funding from the European Community's Seventh Framework Programme (FP7-INFRASTRUCTURES-2008-1) under grant agreement no 228334 (LASERLAB-EUROPE, The Integrated Initiative of European Laser Research Infrastructures II).

\section{References}

Arridge, S.R., Cope, M., Delpy, D.T., 1992. The theoretical basis for the determination of optical pathlengths in tissue: temporal and frequency analysis. Physics in Medicine and Biology 37, 1531-1560.

Baranyai, L., Zude, M., 2009. Analysis of laser light propagation in kiwifruit using backscattering imaging and Monte Carlo simulation. Computers and Electronics in Agriculture 69, 33-39.

Birth, G.S., Hecht, H.G., 1987. The physics of near-infrared reflectence. In: Williams, P., Norris, K. (Eds.), Near-Infrared Technology in the Agricultural and Food Industries. American Association of Cereal Chemists Inc., St. Paul, MN, pp. 1-15 
Contini, D., Martelli, F., Zaccanti, G., 1997. Photon migration through a turbid slab described by a model based on diffusion approximation. 2. Theory. Applied Optics 36, 4587-4599.

Cubeddu, R., Pifferi, A., Taroni, P., Torricelli, A., Valentini, G., 1996a. Experimental test of theoretical models for time-resolved reflectance. Medical Physics 23, 1625-1633.

Cubeddu, R., Pifferi, A., Taroni, P., Torricelli, A., Valentini, G., Ruiz-Altisent, M. Valero, C., Ortiz, C., 1999. Non-destructive measurements of the optical properties of fruits by means of time-resolved reflectance. In: Chance, B., Alfano, R.R., Tromberg, B.J., (Eds.), Optical Tomography and Spectroscopy of Tissue, III 3597, SPIE Press, Bellingham, pp. 445-449.

Eccher Zerbini, P., Vanoli, M., Grassi, M., Rizzolo, A., Fibiani, M., Cubeddu, R., Pifferi, A., Spinelli, L., Torricelli, A., 2006. A model for the softening of nectarines based on sorting fruit at harvest by time-resolved reflectance spectroscopy. Postharvest Biology and Technology 39, 223-232.

Gamon, J.A., Serrano, L., Surfus, J.S., 1997. The photochemical reflectance index: an optical indicator of photosynthetic radiation use efficiency across species, functional types, and nutrient levels. Oecologia 112, 492-501.

Goncalves, B., Silva, A.P., Moutinho-Pereira, J., Bacelar, E., Rosa, E., Meyer, A.S., 2007 Effect of ripeness and postharvest storage on the evolution of colour and anthocyanins in cherries (Prunus avium L.). Food Chemistry 103, 976-984.

Herold, B., Truppel, I., Zude, M., Geyer, M., 2005. Spectral measurements on 'Elstar' apples during fruit development on the tree. Biosystems Engineering 91, 173182.

Jacques, S.L., 1989. Time-resolved reflectance spectroscopy in turbid tissues. IEEE Transactions on Biomedical Engineering 36, 1155-1161.

Karlidag, H., Ercisli, S., Sengul, M., Tosun, M., 2009. Physico-chemical diversity in fruits of wild-growing sweet cherries (Prunus avium L.). Biotechnology \& Biotechnological Equipment 23, 1325-1329.

Knee, M., 1972. Anthocyanin, carotenoid, and chlorophyll changes in the peel of Cox's Orange Pippin apples during ripening on and off the tree. Journal of Experimental Botany 23, 184-196.

Kovacs, E., Muskovics, G., Perlaki, R., 2009. Relationship of colour and other quality parameters of sweet cherry during development and ripening. Acta Alimentaria $38,415-426$

Manganaris, G.A., Ilias, I.F., Vasilakakis, M., Mignani, I., 2007. The effect of hydrocooling on ripening related quality attributes and cell wall physicochemical properties of sweet cherry fruit (Prunus avium L.). International Journal of Refrigeration-Revue Internationale Du Froid 30, 13861392.

Merzlyak, M.N., Gitelson, A.A., Chivkunova, O.B., Rakitin, V.Y., 1999. Non-destructive optical detection of pigment changes during leaf senescence and fruit ripening. Physiologia Plantarum 106, 135-141.

Molto Garcia, E., Blasco, J., 2009. Machine vision systems for raw material detection. In: Zude, M. (Ed.), Optical Monitoring of Fresh and Processed Agricultural Crops CRC Press, Boca Raton, FL, ISBN: 9781420054026, ISBN-10: 1420054023, 576p.

Nagata, M., Yamashita, I. 1992. Simple method for simultaneous determination of chlorophyll and carotenoids in tomato fruit. Journal of the Japan Society Food Science and Technology - Nippon Shokuhin Kagaku Kogaku Kaishi 39, 925-928.
Nicolai, B.M., Beullens, K., Bobelyn, E., Peirs, A., Saeys, W., Theron, K.I., Lammertyn, J., 2007. Nondestructive measurement of fruit and vegetable quality by means of NIR spectroscopy: a review. Postharvest Biology and Technology 46, 99-118.

Oheim, M., Schapper, P., 2005. Non-linear evanescent-field imaging. Journal of Physics D - Applied Physics 38, R185-R197.

Ozaki, Y., McClure, W.F., Christy, A.A., 2006. Near-Infrared Spectroscopy in Food Science and Technology. John Wiley \& Sons, Hoboken.

Patterson, M.S., Chance, B., Wilson, B.C., 1989. Time resolved reflectance and transmittance for the non-invasive measurement of tissue optical properties. Applied Optics 28, 2331-2336

Peirs, A., Schenk, A., Nicoläl, B.M., 2005. Effect of natural variability among apples on the accuracy of VIS-NIR calibration models for optimal harvest date predictions. Postharvest Biology Technology 35, 1-13.

Penuelas, J., Baret, F., Filella, I., 1995. Semiempirical indexes to assess carotenoids chlorophyll-a ratio from leaf spectral reflectance. Photosynthetica 31, 221-230.

Pflanz, M., Zude, M., 2008. Spectrophotometric analyses of chlorophyll and single carotenoids during fruit development of tomato (Solanum lycopersicum L.) by means of iterative multiple linear regression analysis. Applied Optics 47, 59615970 .

Richardson, A.D., Duigan, S.P., Berlyn, G.P., 2002. An evaluation of noninvasive methods to estimate foliar chlorophyll content. New Phytologist 153, 185-194.

Serrano, M., Guillen, F., Martinez-Romero, D., Castillo, S., Valero, D., 2005. Chemical constituents and antioxidant activity of sweet cherry at different ripening stages. Journal of Agricultural and Food Chemistry 53, 2741-2745.

Serrano, M., Diaz-Mula, H.M., Zapata, P.J., Castillo, S., Guillen, F., Martinez-Romero, D., Valverde, J.M., Valero, D., 2009. Maturity stage at harvest determines the fruit quality and antioxidant potential after storage of sweet cherry cultivars. Journal of Agricultural and Food Chemistry 57, 3240-3246.

Solovchenko, A.E., Chivkunova, O.B., Merzlyak, M.N., Gudkovsky, V.A. 2005 Relationships between chlorophyll and carotenoid pigments during on- and off-tree ripening of apple fruit as revealed non-destructively with reflectance spectroscopy. Postharvest Biology and Technology 38, 9-17.

Torricelli, A., 2009. Determination of optical properties in turbid media: timeresolved approach. In: Zude, M. (Ed.). Optical Monitoring of Fresh and Processed Agricultural Crops, CRC Press, Boca Raton, FL, ISBN: 9781420054026, ISBN-10: $1420054023,576 \mathrm{p}$.

Tsuchikawa, S., Hamada, T., 2004. Application of time-of-flight near infrared spectroscopy for detecting sugar and acid contents in apples. Journal of Agricultural and Food Chemistry 52, 2434-2439.

Zude, M., 2003. Comparison of indices and multivariate models to non-destructively predict the fruit chlorophyll by means of visible spectrometry in apple fruit. Analytica Chimica Acta 481, 119-126.

Zude, M., Birlouez-Aragon, I., Paschold, P.J., Rutledge, D.N., 2007. Non-invasive spectrophotometric sensing of carrot quality from harvest to consumption. Postharvest Biology and Technology 45, 30-37.

Zude, M., Spinelli, L., Torricelli, A., 2008. Approach for non-destructive pigment analysis in model liquids and carrots by means of time-of-flight and multiwavelength remittance readings. Analytica Chimica Acta 623, 204-212. 